\title{
Viscoelastic drop deformation in simple shear flow investigated by the front tracking method
}

\author{
C. Chung ${ }^{1}$, M. A. Hulsen ${ }^{2}$, K. H. Ahn ${ }^{1} \&$ S. J. Lee ${ }^{1}$ \\ ${ }^{I}$ School of Chemical and Biological Engineering, \\ Seoul National University, Seoul, Korea \\ ${ }^{2}$ Mechanical Engineering, Eindhoven University of Technology, \\ Eindhoven, Netherlands
}

\begin{abstract}
The two-dimensional deformation of immiscible drop in simple shear flow was investigated using the front tracking method. Interface particles were traced by Runge-Kutta $2^{\text {nd }}$ order method and the boundary immersed method was used for calculation of surface tension force at the global mesh. Isothermal, incompressible and creeping flow was assumed. The main purpose of this research is to analyze the effect of viscosity ratio and elasticity on the drop deformation. Oldroyd-B model was used as a constitutive equation with stabilizing schemes such as DEVSS-G/SUPG and matrix logarithm. As for the Newtonian drop deformation in the Newtonian matrix, there was no breakup until $C a=1$ when the viscosity ratio was one. And the damped oscillation was observed when the viscosity ratio was not unity. The effect of elasticity on the drop deformation was also investigated. As De increased, the drop was more deformed and orientation angle declined to the shear direction.

Keywords: drop deformation, viscosity ratio, elasticity, front tracking method, immersed boundary method, DEVSS-G/SUPG, matrix logarithm.
\end{abstract}

\section{Introduction}

The study of drop deformation in simple shear flow provides an insight for understanding the physics of complex flows. Numerical analysis is required for analyzing applications including polymer processing and multi-phase flow in micro-channels. There have been several studies on the deformation of a drop in shear flow since the pioneering works of Taylor [1,2]. Also numerical approaches $[3,4]$ for the same problem showed good agreements with experiments for 
Newtonian fluids. As for viscoelastic fluids, however, there seems to be a gap to reach a general consent among numerical solutions [5-7]. Here, the first attempt of the front tracking method was made to describe the deformation of the viscoelastic drop in the viscoelastic medium under shear flow.

\section{Mathematical formulation}

To understand the deformation of the interface in the viscoelastic multi-phase fluid, the viscoelastic instability and the capillary instability should be resolved simultaneously. Here, the numerical schemes such as DEVSS-G [8], SUPG [9] and matrix logarithm [10] were applied to stabilize governing equations. And Lagrangian particles (markers) on the interface were traced with Runge-Kutta $2^{\text {nd }}$ order method.

\subsection{Front tracking method}

Front tracking method is the method which tracks the position of the Lagrangian mesh (front mesh) presented as interface in the Eulerian mesh (global mesh) where real calculation is conducted using the values from the nodes of the Eulerian mesh [11]. Established method for the calculation of normal vector and curvature was used to fit a polynomial for the interface. This method requires the information on the position of the markers nearby, where the quadratic interpolation for the position of the interface was good enough for accurate results [12]. Besides this method, another approach for the calculation of surface tension using the tangential vector of the front element was developed [13]. This approach is not only simple but also very accurate since total force on any closed surface is calculated as zero. Here, the surface tension at the interface was calculated according to this method. Using Frenet-Serret theorem [14], the surface tension at the interface, $f_{e}$ is as below:

$$
f_{e}=\int_{\Delta s} \sigma \kappa \mathbf{n} \delta d s=\int_{A}^{B} \sigma \frac{d \mathbf{t}}{d s} d s=\sigma\left(\mathbf{t}_{B}-\mathbf{t}_{A}\right)
$$

where $\sigma$ is the surface tension coefficient, $\delta$ is the Dirac delta function and $\Delta s$ is the average length of the segment of front element. $\mathbf{n}, \mathbf{t}$ are the outward normal vector and tangential vector at the node of front mesh, $\kappa$ is the twice mean curvature of the interface. Though the surface tension is calculated at the front mesh, it is necessary to transfer the values of the front mesh to the global mesh. The interchange of the information between two meshes is possible using immersed boundary method $[15,16]$. In this method, the information between front mesh and global mesh can be transferred using Dirac delta function as follows:

$$
\begin{aligned}
& F_{i j}(\mathbf{x})=\sum_{l} f_{l}(\mathbf{x}) \delta\left(\mathbf{x}-\mathbf{x}_{l}\right) \Delta s_{l}, \\
& f_{l}(\mathbf{x})=\sum_{i j} h_{x} h_{y} F_{i j}(\mathbf{x}) \delta\left(\mathbf{x}-\mathbf{x}_{l}\right),
\end{aligned}
$$

where $h$ is the size of the global element, $F_{i j}(\mathbf{x})$ and $f_{l}(\mathbf{x})$ are the values at the node of global mesh and front mesh, respectively. For two dimensional case, delta function is defined as below: 


$$
\delta(\mathbf{x})=d(x) d(y)
$$

According to Peskin [15]

$$
d(r)=\left\{\begin{array}{cl}
\frac{1+\cos \left(\frac{\pi r}{2 h}\right)}{4 h} & |r| \leq 2 h . \\
0 & |r|>2 h
\end{array}\right.
$$

In this research, the characteristic length $h$ was set to the minimum of the diagonal length of the global element.

Markers representing the interface are positioned with equal distance at the initial state. However, the size of the front element changes as the calculation proceeds since the deformation of the interface changes with time and position. Because large variation of the sizes of front elements would exert a bad influence on the solution, a remeshing algorithm for maintaining the size of the front element in a certain range is necessary. In other words, addition or deletion of the front element should be conducted in case of need. In this research, the length of the front element, $\Delta s_{l}$ was kept from $20 \%$ to $50 \%$ with respect to $h$.

\subsection{Governing equations}

Governing equations for the Newtonian fluid consist of momentum equation and continuity equation. When viscoelasticity is considered, the constitutive equation for the polymer stress should be added. Here, Oldroyd-B model was used as a constitutive equation. Isothermal, incompressible and creeping flow was assumed. To stabilize the calculation, DEVSS-G scheme [8] and SUPG scheme [9] were adopted to the governing equations. Also, matrix logarithm [10] was applied to the constitutive equation.

$$
\begin{aligned}
& \frac{\mathbf{s}^{n+1}-\mathbf{s}^{n}}{\Delta t}+\mathbf{u}^{n} \cdot \nabla \mathbf{s}^{n+1}=\dot{\mathbf{S}}^{n} \\
& \mathbf{G}^{n+1}-\left(\nabla \mathbf{u}^{n+1}\right)^{T}=0 \\
& -\nabla p^{n+1}+\nabla \cdot \eta\left(\nabla \mathbf{u}^{n+1}+\left(\nabla \mathbf{u}^{n+1}\right)^{T}\right)+\eta\left(\beta_{s}-1\right) \nabla \cdot\left(\left(\mathbf{G}^{n+1}\right)^{T}+\mathbf{G}^{n+1}\right) \\
& +\nabla \cdot \boldsymbol{\tau}\left(\mathbf{s}^{n+1}\right)+\int_{\Delta s} \sigma \kappa \mathbf{n} \delta d s=0
\end{aligned}
$$

$$
\nabla \cdot \mathbf{u}^{n+1}=0
$$

Here, $\mathbf{u}$ is the velocity, $p$ the pressure, $\eta$ the solution viscosity and $\lambda$ the relaxation time of polymer.

$$
\begin{aligned}
& \dot{\mathbf{s}}=\sum_{i=1}^{2}\left(2 L_{i i}+\frac{f_{i}}{c_{i}}\right) \mathbf{n}_{i} \mathbf{n}_{i}+\sum_{\substack{i=1 \\
i \neq j}}^{2} \sum_{j=1}^{2} \frac{s_{i}-s_{j}}{c_{i}-c_{j}}\left(c_{j} L_{i j}+c_{i} L_{j i}\right) \mathbf{n}_{i} \mathbf{n}_{j}, \\
& \mathbf{f}=-\frac{1}{\lambda}(\mathbf{c}-\mathbf{I})
\end{aligned}
$$

where $\dot{\mathbf{S}}$ and $\mathbf{f}$ are the transformed matrix of $\dot{\mathbf{s}}$ and $f$ into the global frame. More details for the matrix logarithm were referred to the literature [10]. I is the 
identity tensor and the relation between the conformation tensor, $\mathbf{c}$ and the extra stress tensor, $\tau$ is as follows:

$$
\boldsymbol{\tau}=\frac{\eta \beta_{p}}{\lambda}(\mathbf{c}-\mathbf{I}) .
$$

The polymer contribution to the viscosity, $\beta_{p}$ is defined as:

$$
\beta_{p}=\frac{\eta_{p}}{\eta_{p}+\eta_{s}}=\frac{\eta_{p}}{\eta} .
$$

$\eta_{p}$ and $\eta_{s}$ mean the polymer viscosity and the solution viscosity, respectively. In this research, $\beta_{p}$ was set to $8 / 9$. The problem was defined with a computational domain of width equal to 4 and height equal to 12 . The initial drop was positioned at the center of the domain, coordinates $(2,6)$ with a radius, $a$ as 0.5 . Center region was refined as a structured type for the accurate calculation of the interface (figure 1). For the simulation of large deformation or viscoelastic fluids, the mesh was more refined with structured elements as in UC type. More details on the meshes were shown in table 1. Side walls moved with a constant velocity to make simple shear flow with $\dot{\gamma}=1$. And the steady condition or periodic condition was imposed at the inlet.

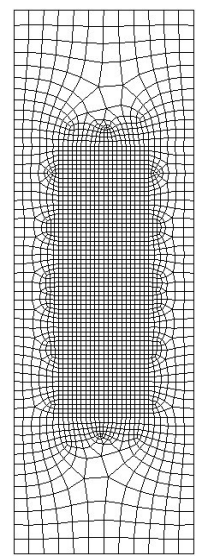

(a) UM1

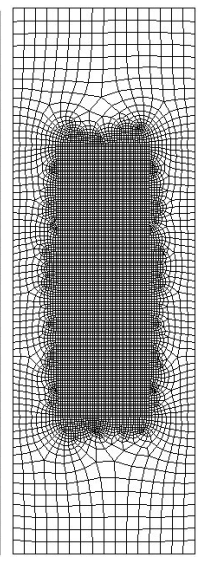

(b) UM2

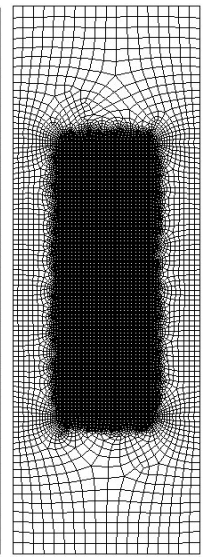

(c) UM3

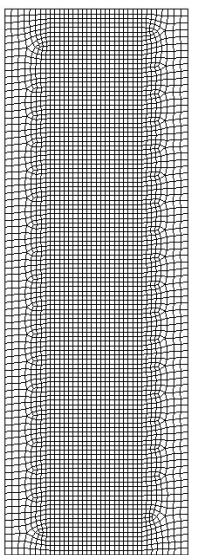

(d) UC1

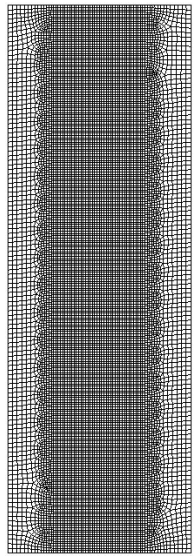

(e) UC2

Figure 1: Mesh configuration.

Table 1: $\quad$ Mesh information.

\begin{tabular}{lrrrl}
\hline Name & Elements & Nodes & \multicolumn{1}{c}{ DOF } & $\Delta x_{\min }=\Delta y_{\min }$ \\
\hline UM1 & 2,300 & 9,333 & 47,070 & 0.1 \\
UM2 & 7,935 & 31,909 & 160,058 & 0.05 \\
UM3 & 26,868 & 107,689 & 539,102 & 0.025 \\
\hline UC1 & 4,032 & 16,361 & 82,510 & 0.1 \\
UC2 & 14,494 & 58,353 & 292,902 & 0.05 \\
\hline
\end{tabular}




\section{Results and discussion}

Dimensionless numbers in this problem are capillary number and Deborah number;

$$
\begin{gathered}
C a=\frac{\eta_{m} \dot{\gamma} a}{\sigma}, \\
D e=\lambda \dot{\gamma} .
\end{gathered}
$$

As $C a$ means the ratio between the surface tension force and viscous force, $\eta_{m}$ is the viscosity of the outside matrix and $\sigma$ is the surface tension coefficient. $\lambda$ is the relaxation time of the fluid. To characterize the shape of deformed drop, the deformation parameter, $D$ is defined as $D=(L-B) /(L+B)$, where $L$ and $B$ are two principal directions of deformed shape, respectively. And the orientation angle is defined as longitudinal direction with respect to the shear direction, as illustrated in figure 2 .

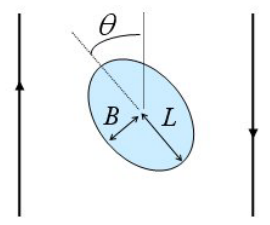

Figure 2: Characterization of the deformed drop.

In order to verify our algorithm, the steady solutions for the Newtonian drop in the Newtonian matrix were compared with previous results (figure 3). As $\mathrm{Ca}$ increases, $D$ increases and orientation angle decreases. As the mesh is more refined, $D$ value converges to the prediction of boundary integral method [17]. And other results of VOF [5] and of diffuse interface method [6] were in line with our results for the small deformation. Though the critical Capillary number, $C a_{c}$ beyond which the drop is not capable of sustaining a stationary shape is known to be around 0.875 [17], here, the steady drop was obtained until $C a=1$ when the viscosity ratio, $\alpha$ was one. The major difference between the reference [17] and ours is the size of the computational domain with respect to the drop size and inlet condition. Though there was no remarkable difference whether periodic condition or steady condition was imposed since the flow domain is large enough comparing with drop size, the periodic effect could not be negligible if small computational domain was used as in reference [17]. With imposing periodic boundary condition at small domain, Zhou and Pozrikidis [17] solved the problem for periodically packed drops closely. While, the problem for the single drop was solved with steady inlet condition in this research. Considering hindering effect of the periodic condition on the drop deformation [18], the difference for $C a_{c}$ could be reasonable. 


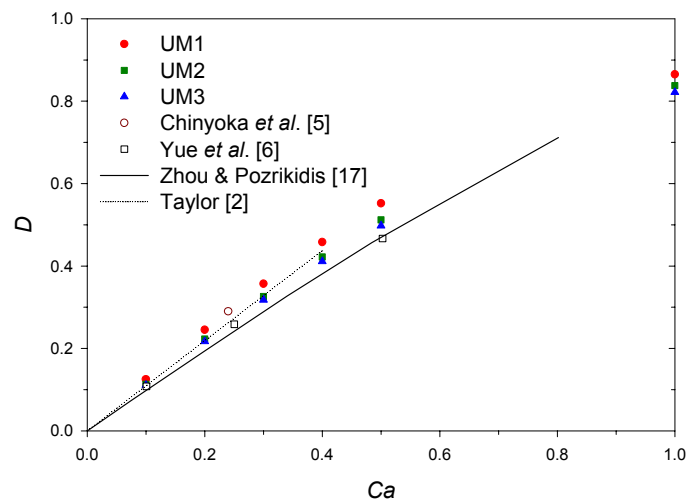

(a) Deformation parameter

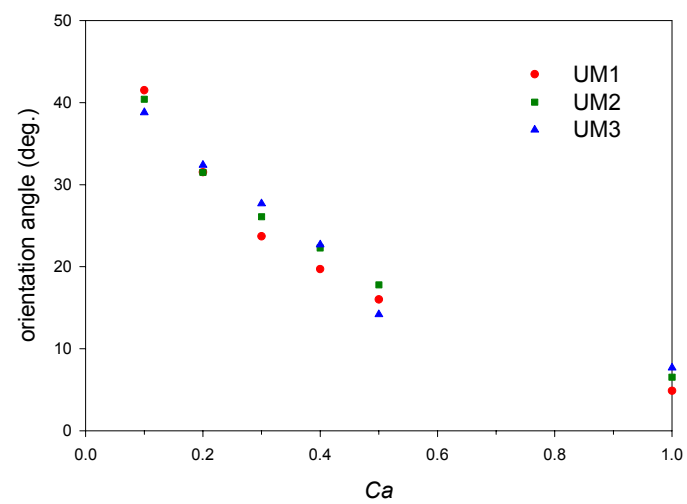

(b) Orientation angle

Figure 3: $\quad$ Steady drop deformation with increasing $C a$.

Shear flow is a mixture of stretching and rotation [19]. Both drop deformation and the rotation of interface are progressed simultaneously. The orientation angle decreases while the initial drop changes to the elongated shape as time passes. When the viscosity ratio was lower than unity, the elongated drop showed somewhat higher orientation angle at early stage because of higher mobility of the drop as shown in figure 4 (a). Then, the drop was more elongated due to high shear force while orientation angle was getting decreased to minimize viscous dissipation [20]. If the orientation angle was close to zero like figure 4 (c), the capillary force became higher than shear force at the head of elongated drop. Hence the drop shows a tendency of coming back to the initial shape. And the shrunk drop was deformed again by shear force. Repeating these behaviours, a damped oscillation in $D$ was observed. Basically, oscillatory behaviour comes from different viscosity ratio. At the experiments of drop with zero interfacial tension when the viscosity ratio was 21 , the rotation of the drop was reported 
[21]. The same phenomenon was reproduced using perturbation theory [22] even though it showed some discrepancy with experimental results [21]. The simulation of three dimensional drop at similar condition reproduced periodic behaviour [23]. At the zero interfacial condition, namely $C a=\infty$ and $\alpha=10$, the periodic oscillation in $D$ was reproduced with our algorithm. Therefore, oscillation is attributed to different viscosity ratio. The damping behaviour may be originated from the surface tension, in other words $C a$.

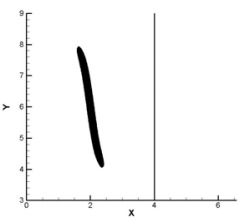

(a) $\mathrm{t}=10$

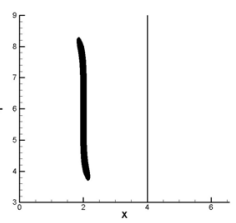

(b) $\mathrm{t}=20$

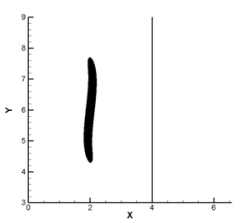

(c) $\mathrm{t}=30$

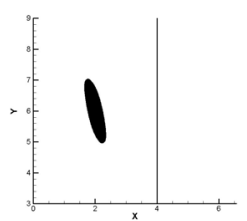

(d) $\mathrm{t}=40$

Figure 4: Oscillatory motion of the drop (UM2, $C a=1, \alpha=0.01$ ).

Damped oscillation behaviour in $D$ was obvious in figure 5 when $\alpha$ was 10 . At the same viscosity ratio, damped oscillation in $D$ was reported using boundary integral method independent of $\mathrm{Ca}$ [17]. When $\alpha<1$, the smaller the viscosity ratio, the shorter the frequency of the oscillation, while vice versa for $\alpha>1$ as shown in figure 5. The larger drop viscosities and matrix lead to larger damping rate, which means that it would take more time to reach steady state.

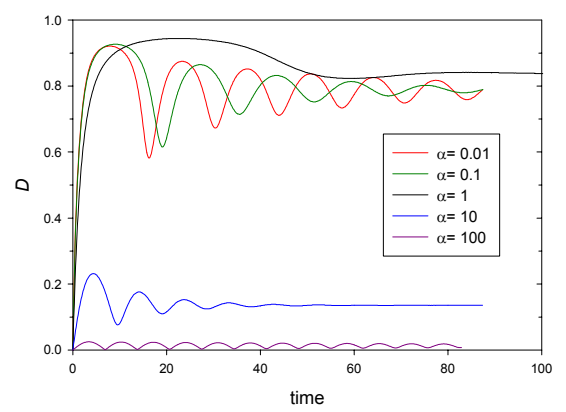

Figure 5: Deformation parameter according to viscosity ratio (UM2, $C a=1$ ).

When compared with the Newtonian drop, an interesting phenomenon was observed in the viscoelastic case. It was reported that the viscoelastic drop was less deformed than the Newtonian case [5]. As De increases, $D$ increases and orientation angle decreases as shown in figure 6. In this case, both drop and matrix are viscoelastic. Though relaxation times should be defined for both fluids, the same relaxation times were used for both fluids in this study as a preliminary step. 


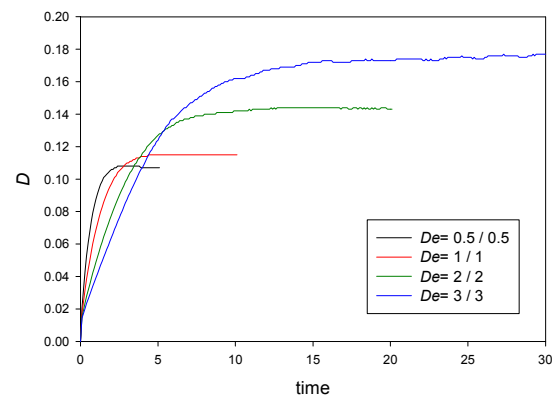

(a) Deformation parameter

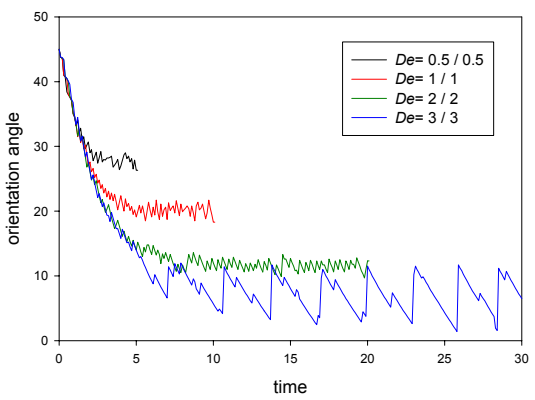

(b) Orientation angle

Figure 6: Transient viscoelastic drop deformation in the viscoelastic flow (UC2).

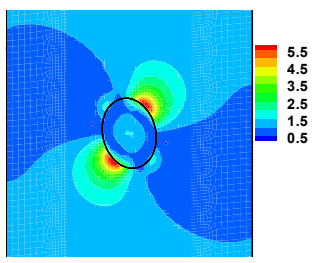

(a) $c_{x x}$

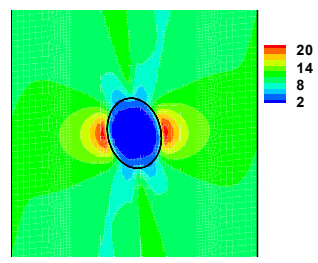

(b) $c_{y y}$

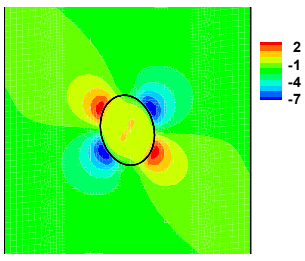

(c) $c_{x y}$

Figure 7: Distribution of conformation tensor (UC2, $D e=2 / 2, C a=0.1$ ).

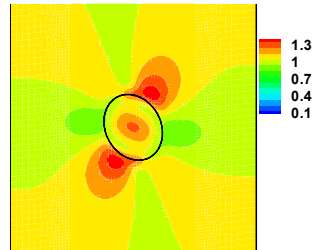

(a) $c_{x x}$

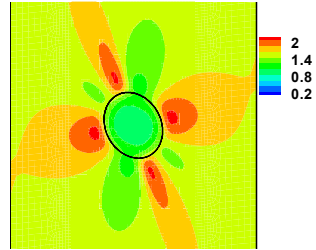

(b) $c_{y y}$

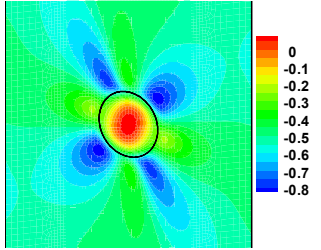

(c) $c_{x y}$

Figure 8: Distribution of conformation tensor (UC2, $D e=0.5 / 0.5, C a=0.1$ ).

At higher $D e$, the drop was more elongated due to higher $c_{y y}$ (yy-component of the conformation tensor) at the waist of the drop than $c_{x x}$ at the head of the drop, and the orientation angle was more tilted to the shear direction than the Newtonian case because of $c_{x y}$ distribution near the drop (figure 7).

At lower $D e$, however, the drop was less deformed than the Newtonian case due to the additional $c_{y y}$ distribution at the head of the drop. And the orientation 
angle was less tilted to the shear direction due to the opposite sign of $c_{x y}$ near the drop in comparison with higher De case.

\section{Conclusions}

Deformation of two dimensional drop in simple shear flow was investigated. Front tracking method showed stable results for interface position. In the Newtonian case, mesh convergence was accomplished. Damped oscillation behaviour in the prediction of drop deformation was reproduced when the viscosities of drop and matrix were different. With stabilizing schemes such as DEVSS-G/SUPG and matrix logarithm, numerical simulation for viscoelastic fluids was also successful. One of the main contributions is that this is the first application of front tracking method to the viscoelastic problem. Comparing with other results, we would get the reliability of our solutions. In this research, the viscoelastic drop showed an interesting phenomenon due to the distribution of conformation tensor near the drop. As $D e$ increases, the drop more deforms and inclines to the shear direction. Major contribution on the deformation of drop seems to come from the elasticity of fluid outside than from inside.

\section{Acknowledgements}

This work was supported by the Korea Research Foundation Grant funded by the Korean Government (MOEHRD) (KRF-2005-213-D00033). The authors wish to acknowledge the National Research Laboratory Fund (M10300000159) of the Ministry of Science and Technology in Korea.

\section{References}

[1] Taylor, G.I, The viscosity of a fluid containing small drops of another fluid. Proc. R. Soc. London, Ser. A, 138, 41-48, 1932.

[2] Taylor, G.I., The formation of emulsions in definable fields of flow. Proc. R. Soc. London, Ser. A, 146, 501-523, 1934.

[3] Li, J., Renardy, Y.Y. \& Renardy M., Numerical simulation of breakup of a viscous drop in simple shear flow through a volume-of-fluid method. Phys. Fluids, 12(2), 269-282, 2000.

[4] Cristini, V., Blawzdziewicz, J. \& Loewenberg M., An adaptive mesh algorithm for evolving surfaces: simulations of drop breakup and coalescence. J. Comput. Phys., 168, 445-463, 2001.

[5] Chinyoka, T., Renardy, Y.Y., Renardy, M. \& Khismatullin, D.B., Twodimensional study of drop deformation under simple shear for Oldroyd-B liquids. J. Non-Newtonian Fluid Mech., 130, 45-56, 2005.

[6] Yue, P., Feng, J.J., Liu, C. \& Shen, J., A diffuse-interface method for simulating two-phase flows of complex fluids. J. Fluid Mech., 515, 293$317,2004$. 
[7] Pillapakkam, S.B. \& Singh, P., A level-set method for computing solutions to viscoelastic two-phase flow. J. Comput. Phys., 174, 552-578, 2001.

[8] Liu, A.W., Bornside, D.E., Armstrong, R.C. \& Brown, R.A., Viscoelastic flow of polymer solutions around a periodic, linear array of cylinders: comparisons of predictions for microstructure and flow fields. J. NonNewtonian Fluid Mech., 77, 153-190, 1998.

[9] Brooks, N. \& Hughes, T.J.R., Streamline upwind/Petrov-Galerkin formulations for convection dominated flows with particular emphasis on the incompressible Navier-Stokes equations. Comput. Methods Appl. Mech. Eng., 32, 199-259, 1982.

[10] Hulsen, M.A., Fattal, R. \& Kupferman, R., Flow of viscoelastic fluids past a cylinder at high Weissenberg number: Stabilized simulations using matrix logarithms. J. Non-Newtonian Fluid Mech., 127, 27-39, 2005.

[11] Unverdi, S. \& Tryggvason, G., A front-tracking method for viscous, incompressible, multi-fluid flows. J. Comput. Phys., 100, 25-37, 1992.

[12] Zhao, P., Heinrich, J.C. \& Poirier, D.R., Fixed mesh front-tracking methodology for finite element simulations. Int. J. Numer. Meth. Engng., 61, 928-948, 2004.

[13] Shin, S. \& Juric, D., Modelling three-dimensional multiphase flow using a level contour reconstruction method for front tracking without connectivity. J. Comput. Phys., 180, 427-470, 2002.

[14] Millman R.S. \& Parker G.D., Elements of differential geometry (Chapter 2). Local curve theory, Prentice-Hall, pp. 13-48, 1977.

[15] Peskin, C.S., Numerical analysis of blood flow in the heart. J. Comput. Phys., 25, 220-252, 1977.

[16] Mittal, R. \& Iaccarino, G., Immersed boundary methods. Annu. Rev. Fluid Mech., 37, 239-261, 2005.

[17] Zhou, H. \& Pozrikidis, C., The flow of suspensions in channels: single files of drops. Phys. Fluids A, 5(2), 311-324, 1993.

[18] Renardy, Y.Y. \& Cristini, V., Effect of inertia on drop breakup under shear. Phys. Fluids, 13(1), 7-13, 2001.

[19] Macosko, C.W., Viscous liquid (Chapter 2). Rheology: principles, measurement, and applications, VCH Publishers. Inc., pp. 65-108, 1994.

[20] Kennedy, M.R., Pozrikidis, C. \& Skalak, R., Motion and deformation of liquid drops, and the rheology of dilute emulsions in simple shear flow. Computers Fluids, 23, 251-278, 1994.

[21] Torza, S., Cox, R.G. \& Mason, S.G., Particle motions in sheared suspensions XXVII. Transient and steady deformation and burst of liquid drops. J. Colloid Interface Sci., 38, 395-411, 1972.

[22] Rallison, J.M., Note on the time-dependent deformation of a viscous drop which is almost spherical. J. Fluid Mech., 98, 625-633, 1980.

[23] Wetzel, E.D. \& Tucker III, C.L. Droplet deformation in dispersions with unequal viscosities and zero interfacial tension. J. Fluid Mech., 426, 199$228,2001$. 